\title{
GLOBAL EXISTENCE OF WEAK SOLUTIONS TO THE FRIED-GURTIN MODEL OF PHASE TRANSITIONS
}

Abstract. We prove the existence of global in time weak solutions to a three-dimensional system of equations arising in a simple version of the Fried-Gurtin model for the isothermal phase transition in solids. In this model the phase is characterized by an order parameter. The problem considered here has the form of a coupled system of three-dimensional elasticity and parabolic equations. The system is studied with the help of the FaedoGalerkin method using energy estimates.

1. Introduction. In this paper we are concerned with the weak solvability of an initial-boundary value problem for a nonlinear coupled system of three-dimensional elasticity and a relaxation law for a scalar order parameter. The system arises as a simple special case of general phase-field (diffused-interface) theory of isothermal solid-solid phase transitions developed by Fried and Gurtin [2] and Fried and Grach [1].

This theory is based on the balance laws of linear momentum and a microforce with underlying free energy depending on a deformation gradient, a multicomponent order parameter and its gradient. The constitutive dependence on the order parameter and its gradient is in contrast to other well-known phase-field theories of solid-solid transitions due to Falk and Frémond (for references see e.g. the review [8]). In these theories the order parameter is identified with the strain tensor, and the free energy is postulated to be a function of strain, strain gradient, and in nonisothermal situation, also temperature.

In Fried-Gurtin's theory the order parameter represents a new quantity which can have different physical status. In the case of diffusive transitions

2000 Mathematics Subject Classification: 35D05, 35K60, 35Q72, 74B20.

Key words and phrases: Fried-Gurtin model, solid-solid transition, phase-field theory, existence and uniqueness of weak solution, Faedo-Galerkin method. 
it describes atomic arrangements within unit cells of a crystal lattice. For pure martensitic transitions, in which the crystal lattice undergoes a mechanical strain but there are no rearrangements of atoms within cells, the order parameter might be viewed as an artifice that yields a regularization of mechanical equations.

From the mathematical point of view it is important that in this theory the stress tensor is a linear function of strain, the nonlinear effects are only connected with the order parameter.

A special 1-D case of the model and its equilibrium solutions have been analyzed in [9]. Existence of a large number of spatially periodic inhomogeneous solutions has been demonstrated. The stability of these solutions has been investigated. Another variant of the 1-D case and properties of its stationary solution have been studied in [3]. The well-posedness of the 3-D case of the Fried-Gurtin model on a finite time interval has been examined in [5]. The system has been studied with the help of the Leray-Schauder fixed point theorem. The existence and uniqueness of solutions has been established. Using similar methods, the existence and uniqueness of solutions in a simpler 1-D case has been proved in [4].

In this work we study the existence of a global in time weak solution to the system resulting from the Fried-Gurtin model. In the second section we present the model and the system of equations. In the third section we state the assumptions and main results of the paper. In the fourth section we construct a Faedo-Galerkin approximation of the problem and state the existence of solutions to this approximation. Next, we deduce a priori estimates using an energy identity. In the last section we prove the main theorems. The first theorem shows the existence of a weak solution on time interval $[0, T]$. In the second theorem we prove that any such solution can be prolonged to $[0, \infty)$.

2. Model. We now formulate the Fried-Gurtin model in a special case of small strain approximation with the strain represented by the linearized strain tensor $\varepsilon=\varepsilon(\mathbf{u})$ and an unconstrained scalar order parameter $\varphi$ distinguishing between two phases, $a$ and $b$, characterized by $\varphi=0$ and $\varphi=1$.

Let $\Omega \subset \mathbb{R}^{3}$ be a bounded domain with smooth boundary $S$, occupied by a body in a fixed reference configuration. The mechanical evolution of the body is described by a displacement field $\mathbf{u}: \Omega^{T} \rightarrow \mathbb{R}^{3}$ and a scalar order-parameter field $\varphi: \Omega^{T} \rightarrow \mathbb{R}$.

The free energy density $f$ underlying the evolution of the body is assumed to be given as a function of the strain tensor $\varepsilon(\mathbf{u})$, the order parameter $\varphi$, and its spatial gradient $\nabla \varphi$ :

$$
f=f(\varepsilon(\mathbf{u}), \varphi, \nabla \varphi)
$$


where

$$
\varepsilon(\mathbf{u})=\frac{1}{2}\left(\nabla \mathbf{u}+(\nabla \mathbf{u})^{T}\right) .
$$

The relevant Landau-Ginzburg separable form of $f$, which is quadratic in $\varepsilon(\mathbf{u})$ and $\nabla \varphi$, and a nonlinear double-well function in $\varphi$, is specified below.

The model has the form of a nonlinear coupled system of partial differential equations representing the linear momentum balance for the displacement (at constant mass density) and the relaxation law for the order parameter, with some prescribed initial and boundary conditions:

$$
\begin{array}{cc}
\mathbf{u}_{t t}-\nabla \cdot f_{, \varepsilon}(\varepsilon(\mathbf{u}), \varphi, \nabla \varphi)=\mathbf{b} & \text { in } \Omega^{T}, \\
\left.\mathbf{u}\right|_{t=0}=\mathbf{u}_{0},\left.\quad \mathbf{u}_{t}\right|_{t=0}=\mathbf{u}_{1} & \text { in } \Omega, \\
\mathbf{u}=0 & \text { on } S^{T}, \\
\beta \varphi_{t}+f_{, \varphi}(\varepsilon(\mathbf{u}), \varphi, \nabla \varphi)-\nabla \cdot f, \nabla \varphi(\varepsilon(\mathbf{u}), \varphi, \nabla \varphi)=0 & \text { in } \Omega^{T}, \\
\left.\varphi\right|_{t=0}=\varphi_{0} & \text { in } \Omega, \\
\mathbf{n} \cdot f_{, \nabla \varphi}(\varepsilon(\mathbf{u}), \varphi, \nabla \varphi)=0 & \text { on } S^{T},
\end{array}
$$

Here $\mathbf{b}: \Omega^{T} \rightarrow \mathbb{R}^{3}$ is an external body force, $\beta$ is a positive constant called dumping modulus (in general, $\beta$ can depend on $\varepsilon, \varphi, \nabla \varphi, \varphi_{t}$ ), and $\mathbf{n}$ denotes the unit outward normal to $S$. The functions $\mathbf{u}_{0}, \mathbf{u}_{1}, \varphi_{0}$ represent initial conditions for the displacement, velocity and order parameter.

We consider the homogeneous Dirichlet boundary condition for the displacement, assuming that the body is fixed at the boundary $S$, and the homogeneous Neumann boundary condition for the order parameter, which is a typical condition in phase field models.

The free energy. The typical Landau-Ginzburg form of the free energy density is given by

$$
f(\varepsilon(\mathbf{u}), \varphi, \nabla \varphi)=W(\varepsilon(\mathbf{u}), \varphi)+\Psi(\varphi)+\frac{\gamma}{2}|\nabla \varphi|^{2},
$$

with the three terms representing respectively the elastic energy, exchange energy and gradient energy with a constant coefficient $\gamma>0$.

The exchange energy $\Psi(\varphi)$ is a double-well potential with equal minima at $\varphi=0$ and $\varphi=1$, assumed in the standard form

$$
\Psi(\varphi)=\frac{1}{2} \varphi^{2}(1-\varphi)^{2} .
$$

The sum of the last two terms in (3) represents the energy of diffused phase interfaces.

The relevant expressions for the elastic energy $W(\varepsilon, \varphi)$ are given by the following two examples (see [2], [1]): 
EXAMPLE 1.

$$
W(\varepsilon, \varphi)=(1-z(\varphi)) W_{a}(\varepsilon)+z(\varphi) W_{b}(\varepsilon),
$$

where

$$
W_{i}(\varepsilon)=\frac{1}{2}\left(\varepsilon-\bar{\varepsilon}_{i}\right) \cdot \mathbf{A}_{i}\left(\varepsilon-\bar{\varepsilon}_{i}\right), \quad i=a, b,
$$

is the strain energy of phase $i$, and $\bar{\varepsilon}_{i}$ is the natural strain of phase $i$, called the eigenstrain; it is assumed to be constant.

Furthermore, $z: \mathbb{R} \rightarrow[0,1]$ is a smooth scalar interpolation function satisfying:

$$
\begin{array}{ll}
z(\varphi)=0 & \text { for } \varphi \leq 0 \\
0 \leq z(\varphi) \leq 1 & \text { for } \varphi \in(0,1) \\
z(\varphi)=1 & \text { for } \varphi \geq 1
\end{array}
$$

The inequality constraint in (6) is imposed to ensure the physical sense of (5).

The tensors $\mathbf{A}_{i}=\left(\left(\mathbf{A}_{i}\right)_{p q r s}\right)_{p, q, r, s=1,2,3}$ are the fourth order elasticity tensors of isotropic elasticity given by

$$
\mathbf{A}_{i} \varepsilon(\mathbf{u})=\lambda_{i} \operatorname{tr} \varepsilon(\mathbf{u}) \mathbf{I}+2 \mu_{i} \varepsilon(\mathbf{u}), \quad i=a, b,
$$

where $\mathbf{I}=\left(\delta_{p q}\right)_{p, q=1,2,3}$ and $\lambda_{i}, \mu_{i}$ are Lamé constants within the elasticity range, i.e., satisfying $\mu_{i}>0,3 \lambda_{i}+2 \mu_{i}>0$.

The second example is characteristic for diffusive phase transitions in elastic solids (see [2]).

EXAMPLE 2.

$$
W(\varepsilon, \varphi)=\frac{1}{2}(\varepsilon-\bar{\varepsilon}(\varphi)) \cdot \mathbf{A}(\varphi)(\varepsilon-\bar{\varepsilon}(\varphi))
$$

where

$$
\bar{\varepsilon}(\varphi)=(1-z(\varphi)) \bar{\varepsilon}_{a}+z(\varphi) \bar{\varepsilon}_{b}
$$

is the natural stress-free strain depending on the order parameter, $\bar{\varepsilon}_{a}, \bar{\varepsilon}_{b}$ are constant eigenstrains, $z(\cdot)$ is as in Example 1, and $\mathbf{A}(\varphi)$ is the elasticity tensor, in general depending on the order parameter $\varphi$.

For the sake of mathematical analysis in the present paper we shall confine ourselves to the case of homogeneous elasticity, that is, in Example 1: $\mathbf{A}_{a}=\mathbf{A}_{b}=\mathbf{A}$, and in Example 2: $\mathbf{A}(\varphi)=\mathbf{A}$ with $\mathbf{A}$ given by

$$
\mathbf{A} \varepsilon(\mathbf{u})=\lambda \operatorname{tr} \varepsilon(\mathbf{u}) \mathbf{I}+2 \mu \varepsilon(\mathbf{u})
$$

where $\mu>0,3 \lambda+2 \mu>0$.

For further use we record that in the case of homogeneous elasticity, the expressions for the elastic energy and its derivatives with respect to $\varepsilon$ and $\varphi$ are: 
- in Example 1:

(9)

$$
\begin{aligned}
W(\varepsilon, \varphi)= & \frac{1}{2} \varepsilon \cdot \mathbf{A} \varepsilon-\varepsilon \cdot\left[(1-z(\varphi)) \mathbf{A} \bar{\varepsilon}_{a}+z(\varphi) \mathbf{A} \bar{\varepsilon}_{b}\right] \\
& +\frac{1}{2}\left[(1-z(\varphi)) \bar{\varepsilon}_{a} \cdot \mathbf{A} \bar{\varepsilon}_{a}+z(\varphi) \bar{\varepsilon}_{b} \cdot \mathbf{A} \bar{\varepsilon}_{b}\right] \\
W_{, \varepsilon}(\varepsilon, \varphi)= & \mathbf{A} \varepsilon-\left[(1-z(\varphi)) \mathbf{A} \bar{\varepsilon}_{a}+z(\varphi) \mathbf{A} \bar{\varepsilon}_{b}\right] \\
W_{, \varphi}(\varepsilon, \varphi)= & z^{\prime}(\varphi)\left[-\varepsilon \cdot \mathbf{A}\left(\bar{\varepsilon}_{b}-\bar{\varepsilon}_{a}\right)+\frac{1}{2} \bar{\varepsilon}_{b} \cdot \mathbf{A} \bar{\varepsilon}_{b}-\frac{1}{2} \bar{\varepsilon}_{a} \cdot \mathbf{A} \bar{\varepsilon}_{a}\right]
\end{aligned}
$$

- in Example 2:

$$
\begin{aligned}
W(\varepsilon, \varphi) & =\frac{1}{2} \varepsilon \cdot \mathbf{A} \varepsilon-\varepsilon \cdot \mathbf{A} \bar{\varepsilon}+\frac{1}{2} \bar{\varepsilon} \cdot \mathbf{A} \bar{\varepsilon} \\
W_{, \varepsilon}(\varepsilon, \varphi) & =\mathbf{A} \varepsilon-\mathbf{A} \bar{\varepsilon}=\mathbf{A} \varepsilon-\mathbf{A} \bar{\varepsilon}_{a}-z(\varphi) \mathbf{A}\left(\bar{\varepsilon}_{b}-\bar{\varepsilon}_{a}\right) \\
W_{, \varphi}(\varepsilon, \varphi) & =-\varepsilon \cdot \mathbf{A} \bar{\varepsilon}_{, \varphi}+\bar{\varepsilon} \cdot \mathbf{A} \bar{\varepsilon}_{, \varphi} \\
& =z^{\prime}(\varphi)\left(-\varepsilon \cdot \mathbf{A}\left(\bar{\varepsilon}_{b}-\bar{\varepsilon}_{a}\right)+\bar{\varepsilon} \cdot \mathbf{A}\left(\bar{\varepsilon}_{b}-\bar{\varepsilon}_{a}\right)\right)
\end{aligned}
$$

We can rewrite $(9)_{2,3}$ and $(10)_{2,3}$ in the common form

$$
\begin{aligned}
W_{, \varepsilon}(\varepsilon, \varphi) & =\mathbf{A} \varepsilon+z(\varphi) \mathbf{B}-\mathbf{A} \bar{\varepsilon}_{a}, \\
W_{, \varphi}(\varepsilon, \varphi) & =z^{\prime}(\varphi)[\mathbf{B} \cdot \varepsilon+z(\varphi) D+E]
\end{aligned}
$$

where:

- in Example 1:

$$
\begin{aligned}
& \mathbf{B}=-\mathbf{A}\left(\bar{\varepsilon}_{b}-\bar{\varepsilon}_{a}\right) \text { a constant tensor }, \\
& D=0, \\
& E=\frac{1}{2} \bar{\varepsilon}_{b} \cdot \mathbf{A} \bar{\varepsilon}_{b}-\frac{1}{2} \bar{\varepsilon}_{a} \cdot \mathbf{A} \bar{\varepsilon}_{a}=\text { const },
\end{aligned}
$$

- in Example 2:

$$
\begin{aligned}
& \mathbf{B}=-\mathbf{A} \bar{\varepsilon} \text { a constant tensor, } \\
& D=-\mathbf{B} \cdot\left(\bar{\varepsilon}_{b}-\bar{\varepsilon}_{a}\right)=\text { const } \\
& E=-\mathbf{B} \cdot \bar{\varepsilon}_{a} .
\end{aligned}
$$

We point out that in the case of homogeneous elasticity, the functions $W_{, \varepsilon}(\varepsilon, \varphi)$ and $W_{, \varphi}(\varepsilon, \varphi)$ are linear in $\varepsilon$, which essentially simplifies the analysis.

The operator $\mathbf{Q}$. Let $\mathbf{Q}$ be the linear elasticity operator defined by

$$
\mathbf{u} \mapsto \mathbf{Q u}=\nabla \cdot \mathbf{A} \varepsilon(\mathbf{u})=\mu \Delta \mathbf{u}+(\lambda+\mu) \nabla(\nabla \cdot \mathbf{u})
$$

where $\mathbf{A}$ is defined by (8). For further purposes we also recall two additional properties of the operator $\mathbf{Q}$ : 
- $\mathbf{Q}$ is strongly elliptic, i.e.

$$
c\|\mathbf{u}\|_{\mathbf{H}^{2}(\Omega)} \leq\|\mathbf{Q u}\|_{\mathbf{L}_{2}(\Omega)} \quad \text { for } \mathbf{u} \in \mathbf{H}^{2}(\Omega) \cap \mathbf{H}_{0}^{1}(\Omega)
$$

with constant $c$ depending on $\Omega$,

- $\mathbf{Q}$ is selfadjoint on $\mathbf{H}^{2}(\Omega) \cap \mathbf{H}_{0}^{1}(\Omega)$, i.e.

$$
\begin{aligned}
(\mathbf{Q u}, \mathbf{v})_{\mathbf{L}_{2}(\Omega)} & =-\mu(\nabla \mathbf{u}, \nabla \mathbf{v})_{\mathbf{L}_{2}(\Omega)}-(\lambda+\mu)(\nabla \cdot \mathbf{u}, \nabla \cdot \mathbf{v})_{L_{2}(\Omega)} \\
& =(\mathbf{u}, \mathbf{Q} \mathbf{v})_{\mathbf{L}_{2}(\Omega)} \quad \text { for } \mathbf{u}, \mathbf{v} \in \mathbf{H}^{2}(\Omega) \cap \mathbf{H}_{0}^{1}(\Omega) .
\end{aligned}
$$

- $-\mathbf{Q}$ is positive on $\mathbf{H}^{2}(\Omega) \cap \mathbf{H}_{0}^{1}(\Omega)$, i.e.

$$
\begin{aligned}
& (-\mathbf{Q u}, \mathbf{u})_{\mathbf{L}_{2}(\Omega)}=\mu\|\nabla \mathbf{u}\|_{\mathbf{L}_{2}(\Omega)}^{2}+(\lambda+\mu)\|\nabla \cdot \mathbf{u}\|_{L_{2}(\Omega)}^{2} \geq 0 \\
& \quad \text { for } \mathbf{u}, \mathbf{v} \in \mathbf{H}^{2}(\Omega) \cap \mathbf{H}_{0}^{1}(\Omega) .
\end{aligned}
$$

In this notation problem (1), (2) corresponding to the free energy (3), with $\Psi(\varphi)$ given by $(4)$, and $W(\varepsilon, \varphi)$ as in Examples 1, 2 (homogeneous elasticity) takes the form

$$
\begin{array}{ll}
\mathbf{u}_{t t}-\mathbf{Q u}=z^{\prime}(\varphi) \mathbf{B} \nabla \varphi+\mathbf{b} & \text { in } \Omega^{T} \\
\left.\mathbf{u}\right|_{t=0}=\mathbf{u}_{0},\left.\quad \mathbf{u}_{t}\right|_{t=0}=\mathbf{u}_{1} & \text { in } \Omega, \\
\mathbf{u}=0 & \text { on } S^{T}
\end{array}
$$

$$
\begin{array}{ll}
\beta \varphi_{t}-\gamma \Delta \varphi+\Psi^{\prime}(\varphi)+z^{\prime}(\varphi)(\mathbf{B} \cdot \varepsilon(\mathbf{u})+z(\varphi) D+E)=0 & \text { in } \Omega^{T}, \\
\left.\varphi\right|_{t=0}=\varphi_{0} & \text { in } \Omega, \\
\mathbf{n} \cdot \nabla \varphi=0 & \text { on } S^{T},
\end{array}
$$

Notation. Throughout the paper we use the following notations:

$$
\begin{array}{ll}
f_{, i}=\frac{\partial f}{\partial x_{i}}, \quad i=1,2,3, & f_{t}=\frac{d f}{d t} \\
\boldsymbol{\varepsilon}(\mathbf{u})=\left(\varepsilon_{i j}\right)_{i, j=1,2,3}, & z^{\prime}(\varphi)=\frac{d z}{d \varphi}, \\
W_{, \boldsymbol{\varepsilon}}(\varepsilon, \varphi)=\left(\frac{\partial W(\varepsilon, \varphi)}{\partial \varepsilon_{i j}}\right)_{i, j=1,2,3}, & W_{, \varphi}(\varepsilon, \varphi)=\frac{\partial W(\varepsilon, \varphi)}{\partial \varphi},
\end{array}
$$

where space and time derivatives are material. For simplicity, whenever there is no danger of confusion, we omit the arguments $(\varepsilon, \varphi)$. Also the specification of tensor indices is omitted.

Vector and tensor valued mappings are denoted by bold letters. The summation convention over repeated indices is used.

We also use the following notation: for vectors $\mathbf{a}=\left(a_{i}\right), \widetilde{\mathbf{a}}=\left(\widetilde{a}_{i}\right)$, and tensors $\mathbf{B}=\left(B_{i j}\right), \widetilde{\mathbf{B}}=\left(\widetilde{B}_{i j}\right), \mathbf{A}=\left(A_{i j k l}\right)$, we write

$$
\mathbf{a} \cdot \widetilde{\mathbf{a}}=a_{i} \widetilde{a}_{i}, \quad \mathbf{B} \cdot \widetilde{\mathbf{B}}=B_{i j} \widetilde{B}_{i j}, \quad \mathbf{A B}=\left(A_{i j k l} B_{k l}\right)
$$


$\nabla$ and $\nabla \cdot$ denote the gradient and divergence operators with respect to the material point $\mathbf{x} \in \mathbb{R}^{3}$. For the divergence of the tensor field $\varepsilon(\mathbf{x})=\left(\varepsilon_{i j}(\mathbf{x})\right)$ we use the convention of contracting over the last index, i.e.,

$$
\nabla \cdot \boldsymbol{\varepsilon}(\mathbf{x})=\left(\varepsilon_{i j, j}(\mathbf{x})\right) .
$$

We use the standard Sobolev spaces notation, in particular, for simplicity we write

$$
\begin{aligned}
H^{m}(\Omega) & =W_{2}^{m}(\Omega) \quad \text { for } m \in \mathbb{N}, \\
\mathbf{L}_{2}(\Omega) & =\left(L_{2}(\Omega)\right)^{3}, \\
\mathbf{H}^{2}(\Omega) & =\left(H^{2}(\Omega)\right)^{3} .
\end{aligned}
$$

The symbol $(\cdot, \cdot)$ denotes the scalar product in $L_{2}(\Omega)$. For simplicity, we use the same notation to denote the scalar product in $\mathbf{L}_{2}(\Omega)$.

Throughout the paper, $c$ denotes various generic constants, depending on the data of the problem and the domain $\Omega$.

\section{Assumptions and main results}

Assumptions. We study the problem (1), (2) under the following assumptions:

(A1) $\Omega \subset \mathbb{R}^{3}$ is a bounded domain with boundary $S$ of class $C^{2}$. This regularity is needed in the application of the elliptic regularity theory in a construction of bases in the Faedo-Galerkin method.

(A2) The elasticity tensor $\mathbf{A}$ is given by (8) with $\mu>0,3 \lambda+2 \mu>0$.

This ensures the following:

- the coercivity and boundedness of $\mathbf{A}$,

$$
\underline{c}|\varepsilon|^{2} \leq \varepsilon \cdot \mathbf{A} \varepsilon \leq \bar{c}|\varepsilon|^{2} \quad \text { for all } \varepsilon \in \mathbf{S}^{2},
$$

where $\mathbf{S}^{2}$ denotes the set of symmetric second order tensors in $\mathbb{R}^{3}$ onto itself and

$$
\underline{c}=\min \{3 \lambda+2 \mu, 2 \mu\}, \quad \bar{c}=\max \{3 \lambda+2 \mu, 2 \mu\},
$$

- the strong ellipticity of the operator $\mathbf{Q}$ defined by (13).

The last assumption concerns the free energy density:

(A3) The free energy density $f(\varepsilon, \varphi, \nabla \varphi): \mathbf{S}^{2} \times \mathbb{R} \times \mathbb{R}^{3} \rightarrow \mathbb{R}$ has the form (3), with $\Psi: \mathbb{R} \rightarrow \mathbb{R}_{+}$given by (4), and $W(\varepsilon, \varphi): \mathbf{S}^{2} \times \mathbb{R} \rightarrow \mathbb{R}$ given in Examples 1 or 2.

We assume that the function $z: \mathbb{R} \rightarrow[0,1]$ in these examples is at least of class $C^{1}$ satisfying (6) and such that

$$
\left|z^{\prime}(\varphi)\right| \leq c \quad \text { for all } \varphi \in \mathbb{R} .
$$


We note that in view of (4) and (11) it follows from (A3) that there exists a positive constant $c$ such that

$$
\begin{aligned}
\left|\Psi^{\prime}(\varphi)\right| & \leq c\left(|\varphi|^{3}+1\right), \\
\left|W_{, \varphi}(\varepsilon, \varphi)\right| & \leq c(|\varepsilon|+1), \\
\left|W_{, \varepsilon}(\varepsilon, \varphi)\right| & \leq c(|\varepsilon|+1)
\end{aligned}
$$

for all $\varepsilon \in \mathbf{S}^{2}$ and $\varphi \in \mathbb{R}$.

Further, on account of (16) the following lower bounds for the elastic energy hold true:

- in Example 1,

$$
W(\varepsilon, \varphi) \geq \min _{i \in\{a, b\}}\left\{\frac{1}{2} \underline{c}\left|\varepsilon-\bar{\varepsilon}_{i}\right|^{2}\right\}
$$

- in Example 2,

$$
W(\varepsilon, \varphi) \geq \frac{1}{2} \underline{c}\left|\varepsilon-\bar{\varepsilon}_{a}-z(\varphi)\left(\bar{\varepsilon}_{b}-\bar{\varepsilon}_{a}\right)\right|^{2} .
$$

Moreover

$$
\Psi(\varphi) \geq \frac{1}{8} \varphi^{4}-\frac{1}{2}
$$

Thus we can see that the homogeneous part $W(\varepsilon, \varphi)+\Psi(\varphi)$ of $f(\varepsilon, \varphi, \nabla \varphi)$ satisfies the lower bound

$$
W(\varepsilon, \varphi)+\Psi(\varphi) \geq c\left(|\varepsilon|^{2}+|\varphi|^{4}\right)-c \quad \text { for all }(\varepsilon, \varphi) \in \mathbf{S}^{2} \times \mathbb{R} .
$$

Consequently,

$$
\begin{aligned}
& f(\varepsilon, \varphi, \nabla \varphi) \geq c_{f}\left(|\varepsilon|^{2}+|\varphi|^{4}+|\nabla \varphi|^{2}\right)-c_{f}^{\prime} \\
& \qquad \text { for all }(\varepsilon, \varphi, \nabla \varphi) \in \mathbf{S}^{2} \times \mathbb{R} \times \mathbb{R}^{3}
\end{aligned}
$$

with constants $c_{f}>0$ and $c_{f}^{\prime} \geq 0$.

This is the main structure assumption that we use in deriving of energy estimates.

Main results. We now state the main results of the paper.

TheOREM 1 (Existence on $(0, T)$ ). Let the assumptions (A1)-(A3) hold. Moreover, let the data satisfy

$$
\begin{aligned}
\mathbf{b} & \in \mathbf{L}_{2}\left(\Omega^{T}\right), \\
\mathbf{u}_{0} & \in \mathbf{H}_{0}^{1}(\Omega), \quad \mathbf{u}_{1} \in \mathbf{L}_{2}(\Omega), \quad \varphi_{0} \in H^{1}(\Omega) .
\end{aligned}
$$

Then there exist functions $(u, \varphi)$ such that

$$
\begin{aligned}
& \mathbf{u} \in L_{\infty}\left(0, T ; \mathbf{H}_{0}^{1}(\Omega)\right), \quad \mathbf{u}_{t} \in L_{\infty}\left(0, T ; \mathbf{L}_{2}(\Omega)\right), \\
& \mathbf{u}_{t t} \in L_{2}\left(0, T ;\left(\mathbf{H}_{0}^{1}(\Omega)\right)^{\prime}\right), \\
& \varphi \in L_{\infty}\left(0, T ; H^{1}(\Omega)\right) \cap L_{2}\left(0, T ; H^{2}(\Omega)\right), \quad \varphi_{t} \in L_{2}\left(\Omega^{T}\right), \\
& \mathbf{u}(0, \cdot)=\mathbf{u}_{0}, \quad \mathbf{u}_{t}(0, \cdot)=\mathbf{u}_{1}, \quad \varphi(0, \cdot)=\varphi_{0},
\end{aligned}
$$


which satisfy problem (1)-(2) in the following weak sense:

$$
\begin{aligned}
& \int_{0}^{T}\left\langle\mathbf{u}_{t t}, \boldsymbol{\eta}\right\rangle_{\left(\mathbf{H}_{0}^{1}(\Omega)\right)^{\prime}, \mathbf{H}_{0}^{1}(\Omega)} d t+\int_{0}^{T}(\mathbf{A} \boldsymbol{\varepsilon}(\mathbf{u}), \boldsymbol{\varepsilon}(\boldsymbol{\eta}))_{\mathbf{L}_{2}(\Omega)} d t \\
& \quad=\int_{0}^{T}\left(z^{\prime}(\varphi) \mathbf{B} \nabla \varphi+\mathbf{b}, \boldsymbol{\eta}\right)_{\mathbf{L}_{2}(\Omega)} d t \quad \text { for any } \boldsymbol{\eta} \in L_{2}\left(0, T ; \mathbf{H}_{0}^{1}(\Omega)\right), \\
& \int_{0}^{T}\left(\beta \varphi_{t}-\gamma \Delta \varphi+\Psi^{\prime}(\varphi)+W_{, \varphi}(\boldsymbol{\varepsilon}(\mathbf{u}), \varphi), \xi\right)_{L_{2}(\Omega)} d t=0 \\
& \text { for any } \xi \in L_{2}\left(0, T ; L_{2}(\Omega)\right) .
\end{aligned}
$$

Moreover, $(\mathbf{u}, \varphi)$ satisfies the a priori estimates

$$
\begin{aligned}
\|\mathbf{u}\|_{L_{\infty}\left(0, T ; \mathbf{L}_{2}(\Omega)\right)}+\|\boldsymbol{\varepsilon}(\mathbf{u})\|_{L_{\infty}\left(0, T ; \mathbf{L}_{2}(\Omega)\right)}+\|\varphi\|_{L_{2}\left(0, T ; L_{4}(\Omega)\right)} & \\
+\|\nabla \varphi\|_{L_{\infty}\left(0, T ; \mathbf{L}_{2}(\Omega)\right)}+\left\|\varphi_{t}\right\|_{L_{2}\left(\Omega^{T}\right)} & \leq c_{0}, \\
\|\varphi\|_{L_{\infty}\left(0, T ; H^{1}(\Omega)\right)} & \leq c_{1}, \\
\|\varphi\|_{L_{2}\left(0, T ; H^{2}(\Omega)\right)} & \leq c_{2}(T), \\
\left\|\mathbf{u}_{t t}\right\|_{L_{2}\left(0, T ;\left(\mathbf{H}_{0}^{1}(\Omega)\right)^{\prime}\right)} & \leq c_{3}(T)
\end{aligned}
$$

where

$$
\begin{aligned}
c_{0} & =c\left(\left\|\mathbf{u}_{0}\right\|_{\mathbf{H}^{1}(\Omega)},\left\|\mathbf{u}_{1}\right\|_{\mathbf{L}_{2}(\Omega)},\left\|\varphi_{0}\right\|_{H^{1}(\Omega)},\|\mathbf{b}\|_{L_{1}\left(0, T ; \mathbf{L}_{2}(\Omega)\right)}, c_{f}, c_{f}^{\prime}\right), \\
c_{1} & =c\left(c_{0}, \Omega\right), \\
c_{2}(T) & =c\left(c_{1}\right) T^{1 / 2} \\
c_{3}(T) & =c\left(c_{0},\|\mathbf{b}\|_{\mathbf{L}_{2}\left(\Omega^{T}\right)}\right) T^{1 / 2} .
\end{aligned}
$$

THEOREM 2 (Global existence). Assume the hypotheses of Theorem 1 hold, and

$$
\begin{aligned}
& \mathbf{b} \in L_{1}\left(\mathbb{R}_{+}, \mathbf{L}_{2}(\Omega)\right), \\
& \sup _{k \in \mathbb{N} \cup\{0\}}\|\mathbf{b}\|_{L_{2}\left(k T,(k+1) T ; \mathbf{L}_{2}(\Omega)\right)}<\infty, \\
& \mathbf{u}_{0} \in \mathbf{H}_{0}^{1}(\Omega), \quad \mathbf{u}_{1} \in \mathbf{L}_{2}(\Omega), \quad \varphi_{0} \in H^{1}(\Omega) .
\end{aligned}
$$

Then there exists a global solution $(\mathbf{u}, \varphi)$ to problem $(1)-(2)$ such that

$$
\begin{aligned}
& \mathbf{u} \in L_{\infty}\left(\mathbb{R}_{+} ; \mathbf{H}_{0}^{1}(\Omega)\right), \quad \mathbf{u}_{t} \in L_{\infty}\left(\mathbb{R}_{+} ; \mathbf{L}_{2}(\Omega)\right), \\
& \varphi \in L_{\infty}\left(\mathbb{R}_{+} ; H^{1}(\Omega)\right), \quad \varphi_{t} \in L_{\infty}\left(\mathbb{R}_{+} ; L_{2}(\Omega)\right), \\
& \mathbf{u}(0)=\mathbf{u}_{0}, \quad \mathbf{u}_{t}(0)=\mathbf{u}_{1}, \quad \varphi(0)=\varphi_{0},
\end{aligned}
$$

satisfying the following estimates: 
- estimates uniform in time:

$$
\begin{aligned}
\|\mathbf{u}\|_{L_{\infty}\left(\mathbb{R}_{+} ; \mathbf{H}_{0}^{1}(\Omega)\right)}+\|\varepsilon(\mathbf{u})\|_{L_{\infty}\left(\mathbb{R}_{+} ; \mathbf{L}_{2}(\Omega)\right)}+\|\varphi\|_{L_{\infty}\left(\mathbb{R}_{+} ; L_{4}(\Omega)\right)} & \\
+\|\nabla \varphi\|_{L_{\infty}\left(\mathbb{R}_{+} ; \mathbf{L}_{2}(\Omega)\right)}+\left\|\varphi_{t}\right\|_{L_{2}\left(\mathbb{R}_{+} ; L_{2}(\Omega)\right)} & \leq c_{0}, \\
& \|\varphi\|_{L_{\infty}\left(\mathbb{R}_{+} ; H^{1}(\Omega)\right)} \leq c_{1} .
\end{aligned}
$$

- estimates on finite intervals $(t, t+T)$ where $t \in \mathbb{R}_{+}$and $T>0$ is fixed:

$$
\begin{aligned}
\|\varphi\|_{L_{2}\left(t, t+T ; H^{2}(\Omega)\right)} & \leq c_{2}(T), \\
\left\|\mathbf{u}_{t t}\right\|_{L_{2}\left(t, t+T ;\left(\mathbf{H}_{0}^{1}(\Omega)\right)^{\prime}\right)} & \leq \widetilde{c}_{3}(T),
\end{aligned}
$$

with constants $c_{0}, c_{1}, c_{2}(T)$ specified by $(23)$, and

$$
\widetilde{c}_{3}(T)=c\left(c_{0}, \sup _{k \in \mathbb{N} \cup\{0\}}\|\mathbf{b}\|_{L_{2}\left(k T,(k+1) T ; \mathbf{L}_{2}(\Omega)\right)}\right) T^{1 / 2} .
$$

\section{The Faedo-Galerkin approximation of the problem}

An approximation. To prove Theorems 1 and 2 using the Faedo-Galerkin method we construct bases for the spaces $\mathbf{H}_{0}^{1}(\Omega)$ and

$$
H_{N}^{2}(\Omega)=\left\{w \in H^{2}(\Omega): \mathbf{n} \cdot \nabla w=0 \text { on } S\right\} .
$$

For $j \in \mathbb{N}$, consider the eigenvalue problems

$$
\begin{aligned}
-\mathbf{Q} \mathbf{v}_{j} & =\lambda_{j}^{(1)} \mathbf{v}_{j} & & \text { in } \Omega, \\
\mathbf{v}_{j} & =0 & & \text { on } S,
\end{aligned}
$$

and

$$
\begin{aligned}
-\Delta w_{j} & =\lambda_{j}^{(2)} w_{j} & & \text { in } \Omega, \\
\mathbf{n} \cdot \nabla w_{j} & =0 & & \text { on } S .
\end{aligned}
$$

We recall that, by the elliptic regularity theory for a domain with boundary of class $C^{2}$, the solutions of (24) and (25) satisfy

$$
\mathbf{v}_{j} \in \mathbf{H}^{2}(\Omega), \quad w_{j} \in H^{2}(\Omega) .
$$

We take the family $\left\{\mathbf{v}_{j}\right\}_{j=1,2, \ldots}$ as a basis of $\mathbf{H}_{0}^{1}(\Omega)$ and the family $\left\{w_{j}\right\}_{j=1,2, \ldots}$ as a basis of $H_{N}^{2}(\Omega)$. By the properties of $\mathbf{Q}$ we have

$$
\begin{aligned}
\lambda_{i}^{(1)}\left(\mathbf{v}_{i}, \mathbf{v}_{j}\right) & =\left(\lambda_{i}^{(1)} \mathbf{v}_{i}, \mathbf{v}_{j}\right)=\left(-\mathbf{Q} \mathbf{v}_{i}, \mathbf{v}_{j}\right)=\left(\mathbf{v}_{i},-\mathbf{Q} \mathbf{v}_{j}\right)=\left(\mathbf{v}_{i}, \lambda_{j}^{(1)} \mathbf{v}_{j}\right) \\
& =\lambda_{j}^{(1)}\left(\mathbf{v}_{i}, \mathbf{v}_{j}\right) .
\end{aligned}
$$

These identities show, by the Poincaré-Friedrichs inequality, that the family $\left\{\mathbf{v}_{j}\right\}$ is orthogonal in $\mathbf{H}^{1}(\Omega)$ and $\mathbf{L}^{2}(\Omega)$ scalar products.

Similarly, the family $\left\{w_{j}\right\}_{j=1,2, \ldots}$ satisfies

$$
\begin{aligned}
\lambda_{i}^{(2)}\left(w_{i}, w_{j}\right) & =\left(\lambda_{i}^{(2)} w_{i}, w_{j}\right)=\left(-\Delta w_{i}, w_{j}\right)=\left(\nabla w_{i}, \nabla w_{j}\right) \\
& =\left(w_{i},-\Delta w_{j}\right)=\left(w_{i}, \lambda_{j}^{(2)} w_{j}\right)=\lambda_{j}^{(2)}\left(w_{i}, w_{j}\right) .
\end{aligned}
$$


Hence, by the Poincaré inequality, the family $\left\{w_{j}\right\}_{j=1,2, \ldots}$ is orthogonal in $H^{2}(\Omega), H^{1}(\Omega)$ and $L^{2}(\Omega)$ scalar products.

We can normalize both families in $\mathbf{L}^{2}(\Omega)$ and $L^{2}(\Omega)$ scalar products respectively.

For $m \in \mathbb{N}$ set

$$
\mathbf{V}_{m}=\operatorname{span}\left\{\mathbf{v}_{1}, \ldots, \mathbf{v}_{m}\right\}, \quad W_{m}=\operatorname{span}\left\{w_{1}, \ldots, w_{m}\right\} ;
$$

these are finite-dimensional subspaces of $\mathbf{H}_{0}^{1}(\Omega)$ and $H_{N}^{2}(\Omega)$ respectively.

For any $m \in \mathbb{N}$ we will find a pair of functions $\left(\mathbf{u}^{m}, \varphi^{m}\right)$ in the form

$$
\mathbf{u}^{m}(x, t)=\sum_{i=1}^{m} e_{i}^{m}(t) \mathbf{v}_{i}(x), \quad \varphi^{m}(x, t)=\sum_{i=1}^{m} c_{i}^{m}(t) w_{i}(x)
$$

satisfying for a.e. $t \in[0, T]$,

$$
\begin{aligned}
& \left(\mathbf{u}_{t t}^{m}, \mathbf{v}_{j}\right)+\left(\mathbf{A} \varepsilon\left(\mathbf{u}^{m}\right), \boldsymbol{\varepsilon}\left(\mathbf{v}_{j}\right)\right)=\left(z^{\prime}\left(\varphi^{m}\right) \mathbf{B} \nabla \varphi^{m}+\mathbf{b}, \mathbf{v}_{j}\right), \\
& \beta\left(\varphi_{t}^{m}, w_{j}\right)-\gamma\left(\Delta \varphi^{m}, w_{j}\right)+\left(\Psi^{\prime}\left(\varphi^{m}\right)+W_{, \varphi}\left(\varepsilon\left(\mathbf{u}^{m}\right), \varphi^{m}\right), w_{j}\right)=0, \\
& \quad \text { for } j=1, \ldots, m, \\
& \mathbf{u}^{m}(0)=\mathbf{u}_{0}^{m}, \quad \mathbf{u}_{t}^{m}(0)=\mathbf{u}_{1}^{m}, \quad \varphi^{m}(0)=\varphi_{0}^{m}
\end{aligned}
$$

where $W_{, \varphi}(\varepsilon(\mathbf{u}), \varphi), \mathbf{B}, D, E$ are given by (12).

Furthermore, $\mathbf{u}_{0}^{m}, \mathbf{u}_{1}^{m} \in \mathbf{V}_{m}, \varphi_{0}^{m} \in W_{m}$ are projections of $\mathbf{u}_{0}, \mathbf{u}_{1}, \varphi_{0}$ respectively, satisfying, as $m \rightarrow \infty$,

$$
\begin{array}{ll}
\mathbf{u}_{0}^{m} \rightarrow \mathbf{u}_{0} & \text { strongly in } \mathbf{H}_{0}^{1}(\Omega), \\
\mathbf{u}_{1}^{m} \rightarrow \mathbf{u}_{1} & \text { strongly in } \mathbf{L}_{2}(\Omega), \\
\varphi_{0}^{m} \rightarrow \varphi_{0} & \text { strongly in } H^{1}(\Omega) .
\end{array}
$$

Existence of solutions to the Faedo-Galerkin approximation. Substituting (26) into (27), taking into account that

$$
\begin{aligned}
\left(\mathbf{u}_{t t}^{m}, \mathbf{v}_{j}\right) & =\left(\sum_{i=1}^{m} e_{i, t t}^{m}(t) \mathbf{v}_{i}, \mathbf{v}_{j}\right)=\sum_{i=1}^{m} e_{i, t t}^{m}(t)\left(\mathbf{v}_{i}, \mathbf{v}_{j}\right)=e_{j, t t}^{m}(t), \\
\left(\varphi_{t}^{m}, w_{j}\right) & =\left(\sum_{i=1}^{m} c_{i, t}^{m}(t) w_{i}, w_{j}\right)=\sum_{i=1}^{m} c_{i, t}^{m}(t)\left(w_{i}, w_{j}\right)=c_{j, t}^{m}(t), \\
-\left(\mathbf{A} \varepsilon\left(\mathbf{u}^{m}\right), \mathbf{v}_{j}\right) & =\left(\sum_{i=1}^{m}-e_{i}^{m}(t) \mathbf{A} \varepsilon\left(\mathbf{v}_{i}\right), \mathbf{v}_{j}\right)=\sum_{i=1}^{m} e_{i}^{m}(t)\left(-\mathbf{A} \varepsilon\left(\mathbf{v}_{i}\right), \mathbf{v}_{j}\right) \\
& =\sum_{i=1}^{m} e_{i}^{m}(t)\left(\lambda_{i}^{(1)} \mathbf{v}_{i}, \mathbf{v}_{j}\right)=\lambda_{j}^{(1)} e_{j}^{m}(t)\left(\mathbf{v}_{j}, \mathbf{v}_{j}\right)=\lambda_{j}^{(1)} e_{j}^{m}(t),
\end{aligned}
$$




$$
\begin{aligned}
\left(-\Delta \varphi^{m}, w_{j}\right) & =\left(\sum_{i=1}^{m}-c_{i}^{m}(t) \Delta w_{i}, w_{j}\right)=\sum_{i=1}^{m} c_{i}^{m}(t)\left(-\Delta w_{i}, w_{j}\right) \\
& =\sum_{i=1}^{m} c_{i}^{m}(t)\left(\lambda_{i}^{(2)} w_{i}(x), w_{j}\right)=c_{j}^{m}(t)\left(\lambda_{j}^{(2)} w_{j}(x), w_{j}\right) \\
& =\lambda_{j}^{(2)} c_{j}^{m}(t)
\end{aligned}
$$

we get

$$
\begin{aligned}
& e_{j, t t}^{m}(t)=\lambda_{j}^{(1)} e_{j}^{m}(t)+\left(z^{\prime}\left(\varphi^{m}\right) \mathbf{B} \nabla \varphi^{m}+\mathbf{b}, \mathbf{v}_{j}\right), \\
& \beta c_{j, t}^{m}(t)=-\gamma \lambda_{j}^{(2)} c_{j}^{m}(t)-\left(\Psi^{\prime}\left(\varphi^{m}\right)-W_{, \varphi}\left(\varepsilon\left(\mathbf{u}^{m}\right), \varphi^{m}\right), w_{j}\right), \\
& \quad \text { for } j=1, \ldots, m .
\end{aligned}
$$

Consequently, the system (11) can be examined as a system of first order ordinary differential equations for the coefficients $\left(e_{1}^{m}, \ldots, e_{m}^{m}\right),\left(e_{1, t}^{m}, \ldots, e_{m, t}^{m}\right)$, $\left(c_{1}^{m}, \ldots, c_{m}^{m}\right)$.

The above system for any $m$ has a solution local in time on an interval $\left[0, T_{m}\right], T_{m}>0$. The uniform (in $m$ ) a priori estimates proved below in Lemmas 1 and 2 show that this system has a solution on an interval $[0, T]$, $T>0$.

\section{A priori estimates for the Faedo-Galerkin approximation}

LEMMA 1 (Energy estimate). Assume that (A1)-(A3) hold and the data satisfy

$$
\mathbf{u}_{0} \in \mathbf{H}_{0}^{1}(\Omega), \mathbf{u}_{1} \in \mathbf{L}_{2}(\Omega), \varphi_{0} \in H^{1}(\Omega), \mathbf{b} \in L_{1}\left(0, T ; \mathbf{L}_{2}(\Omega)\right) .
$$

Then a solution $\left(u^{m}, \varphi^{m}\right)$ to the problem (1)-(2) satisfies the estimate

$$
\begin{aligned}
& \left\|\mathbf{u}_{t}^{m}\right\|_{L_{\infty}\left(0, T ; \mathbf{L}_{2}(\Omega)\right)}+\left\|\boldsymbol{\varepsilon}\left(\mathbf{u}^{m}\right)\right\|_{L_{\infty}\left(0, T ; \mathbf{L}_{2}(\Omega)\right)} \\
& \quad+\left\|\varphi^{m}\right\|_{L_{\infty}\left(0, T ; L_{4}(\Omega)\right)}+\left\|\nabla \varphi^{m}\right\|_{L_{\infty}\left(0, T ; \mathbf{L}_{2}(\Omega)\right)}+\left\|\varphi_{t}^{m}\right\|_{L_{2}\left(\Omega^{T}\right)} \leq c_{0}
\end{aligned}
$$

with a constant

$$
c_{0}=c\left(\left\|\mathbf{u}_{0}\right\|_{\mathbf{H}^{1}(\Omega)},\left\|\mathbf{u}_{1}\right\|_{\mathbf{L}_{2}(\Omega)},\left\|\varphi_{0}\right\|_{H^{1}(\Omega)},\|\mathbf{b}\|_{L_{1}\left(0, T ; \mathbf{L}_{2}(\Omega)\right)}, c_{f}, c_{f}^{\prime}\right) .
$$

Proof. We derive the energy identity for system (1)-(2). First, note that, according to $(11)_{1}$ we have

$$
\nabla \cdot W_{, \varepsilon}(\varepsilon(\mathbf{u}), \varphi)=\nabla \cdot \mathbf{A} \varepsilon(\mathbf{u})+z^{\prime}(\varphi) \mathbf{B} \nabla \varphi .
$$

Thus integration by parts shows that $(27)_{1}$ may be rewritten as

$$
\left(\mathbf{u}_{t t}^{m}, \mathbf{v}_{j}\right)+\left(W_{, \boldsymbol{\varepsilon}}\left(\boldsymbol{\varepsilon}\left(\mathbf{u}^{m}\right), \varphi^{m}\right), \boldsymbol{\varepsilon}\left(\mathbf{v}_{j}\right)\right)=\left(\mathbf{b}, \mathbf{v}_{j}\right)
$$

Testing (30) with $\mathbf{u}_{t}^{m}(t)$ (i.e. multiplying by $e_{j}^{m}(t)$ and summing over $j$ from $j=1$ to $j=m$ ) gives

$$
\frac{1}{2} \frac{d}{d t}\left\|\mathbf{u}_{t}^{m}\right\|_{\mathbf{L}_{2}(\Omega)}^{2}+\left(W_{, \boldsymbol{\varepsilon}}\left(\varepsilon\left(\mathbf{u}^{m}\right), \varphi^{m}\right), \boldsymbol{\varepsilon}\left(\mathbf{u}_{t}^{m}\right)\right)=\left(\mathbf{b}, \mathbf{u}_{t}^{m}\right) .
$$


Further testing $(27)_{2}$ with $\varphi_{t}^{m}(t)$ and integrating by parts gives

$$
\begin{aligned}
\beta\left\|\varphi_{t}^{m}\right\|_{L_{2}(\Omega)}^{2}+\left(\Psi^{\prime}\left(\varphi^{m}\right)+W_{, \varphi}\left(\varepsilon\left(\mathbf{u}^{m}\right), \varphi^{m}\right)\right. & \left., \varphi_{t}^{m}\right) \\
& +\frac{\gamma}{2} \frac{d}{d t}\left\|\nabla \varphi^{m}\right\|_{\mathbf{L}_{2}(\Omega)}^{2}=0 .
\end{aligned}
$$

Summing up (31) and (32) we arrive at the energy identity

$$
\begin{aligned}
\frac{1}{2} \frac{d}{d t} \int_{\Omega}\left|\mathbf{u}_{t}^{m}\right|^{2} d x+\frac{d}{d t} \int_{\Omega}\left(W \left(\varepsilon\left(\mathbf{u}^{m}, \varphi^{m}\right)\right.\right. & \left.+\Psi\left(\varphi^{m}\right)+\frac{1}{2} \gamma\left|\nabla \varphi^{m}\right|^{2}\right) d x \\
& +\beta \int_{\Omega}\left(\varphi_{t}^{m}\right)^{2} d x=\int_{\Omega} \mathbf{b} \cdot \mathbf{u}_{t}^{m} d x .
\end{aligned}
$$

Integration of $(33)$ over $(0, t)$ for $t \in(0, T)$ gives

$$
\begin{array}{r}
\frac{1}{2}\left\|\mathbf{u}_{t}^{m}(t)\right\|_{\mathbf{L}_{2}(\Omega)}^{2}+\int_{\Omega} f\left(\varepsilon\left(\mathbf{u}^{m}(t)\right), \varphi^{m}(t), \nabla \varphi^{m}(t)\right) d x+\beta \int_{\Omega}\left(\varphi_{t}^{m}\right)^{2} d x d t \\
=\frac{1}{2}\left\|\mathbf{u}_{1}^{m}\right\|_{\mathbf{L}_{2}(\Omega)}^{2}+\int_{\Omega} f\left(\varepsilon\left(\mathbf{u}_{0}^{m}\right), \varphi_{0}^{m}, \nabla \varphi_{0}^{m}\right) d x+\int_{\Omega^{t}} \mathbf{b} \cdot \mathbf{u}_{t}^{m} d x d t^{\prime}
\end{array}
$$

with $f(\varepsilon(\mathbf{u}), \varphi, \nabla \varphi)$ defined by (3).

Using the structure condition (18) and estimating the last integral in (34) with the help of the Young inequality by

$$
\begin{aligned}
\left|\int_{\Omega^{t}} \mathbf{b} \cdot \mathbf{u}_{t}^{m} d x d t^{\prime}\right| & \leq\left\|\mathbf{u}_{t^{\prime}}\right\|_{L_{\infty}\left(0, t ; \mathbf{L}_{2}(\Omega)\right)}\|\mathbf{b}\|_{L_{1}\left(0, t ; \mathbf{L}_{2}(\Omega)\right)} \\
& \leq \frac{1}{4}\left\|\mathbf{u}_{t^{\prime}}\right\|_{L_{\infty}\left(0, t ; \mathbf{L}_{2}(\Omega)\right)}^{2}+\|\mathbf{b}\|_{L_{1}\left(0, t ; \mathbf{L}_{2}(\Omega)\right)}^{2},
\end{aligned}
$$

we deduce the a priori estimate

$$
\begin{array}{r}
\text { (35) } \frac{1}{4}\left\|\mathbf{u}_{t}^{m}(t)\right\|_{\mathbf{L}_{2}(\Omega)}^{2}+c_{f}\left(\left\|\varepsilon\left(\mathbf{u}^{m}(t)\right)\right\|_{\mathbf{L}_{2}(\Omega)}^{2}+\left\|\varphi^{m}(t)\right\|_{L_{4}(\Omega)}^{4}+\left\|\nabla \varphi^{m}(t)\right\|_{\mathbf{L}_{2}(\Omega)}^{2}\right) \\
+\beta\left\|\varphi_{t}^{m}\right\|_{L_{2}\left(\Omega^{t}\right)}^{2} \leq c_{0} \quad \text { for } t \in(0, T)
\end{array}
$$

with a constant $c_{0}$ depending only on $\left\|\mathbf{u}_{0}\right\|_{\mathbf{H}^{1}(\Omega)},\left\|\mathbf{u}_{1}\right\|_{\mathbf{L}_{2}(\Omega)},\left\|\varphi_{0}\right\|_{H^{1}(\Omega)}$, $\|\mathbf{b}\|_{L_{1}\left(0, t ; \mathbf{L}_{2}(\Omega)\right)}$ and $c_{f}^{\prime}$. This proves the assertion.

Further estimates. Clearly, (29) implies that

$$
\left\|\varphi^{m}\right\|_{\left.L_{\infty}\left(0, T ; H^{1} \Omega\right)\right)} \leq c_{1}
$$

with a constant $c_{1}=C\left(c_{0}, \Omega\right)$. Hence, by the Sobolev imbedding,

$$
\left\|\varphi^{m}\right\|_{L_{\infty}\left(0, T ; L_{6}(\Omega)\right)} \leq c_{1} .
$$

Further, since $\mathbf{u}^{m}=0$ on $S^{T}$, it follows from (29) by Korn's inequality that

$$
\left\|\mathbf{u}^{m}\right\|_{\left.L_{\infty}\left(0, T ; \mathbf{H}^{1} \Omega\right)\right)} \leq c_{1} .
$$


Lemma 2. Let the assumptions of Lemma 1 hold. Then, for $t \in(0, T]$, (39)

$$
\left\|\varphi^{m}\right\|_{L_{2}\left(0, T ; H^{2}(\Omega)\right)} \leq c_{2}(t)
$$

with a constant $c_{2}(t)=c\left(c_{1}\right) t^{1 / 2}$.

Proof. By the definition (25) of the basis $\left\{w_{1}, \ldots, w_{m}\right\}$ identity $(27)_{2}$ implies that

$$
\beta\left(\varphi_{t}^{m}, \Delta w_{j}\right)-\gamma\left(\Delta \varphi^{m}, \Delta w_{j}\right)+\left(\Psi^{\prime}\left(\varphi^{m}\right)+W_{, \varphi}\left(\varepsilon\left(\mathbf{u}^{m}\right), \varphi^{m}\right), \Delta w_{j}\right)=0
$$

for $j=1, \ldots, m$. Multiplying this equality by $c_{j}^{m}(t)$ and summing from $j=1$ to $j=m$ gives

$$
\gamma\left(\Delta \varphi^{m}, \Delta \varphi^{m}\right)=\beta\left(\varphi_{t}^{m}, \Delta \varphi^{m}\right)+\left(\Psi^{\prime}\left(\varphi^{m}\right)+W_{, \varphi}\left(\varepsilon\left(\mathbf{u}^{m}\right), \varphi^{m}\right), \Delta \varphi^{m}\right) .
$$

Hence, integrating with respect to $t$, we have

$$
\begin{array}{rl}
\gamma \int_{0}^{t} \int_{\Omega}\left|\Delta \varphi^{m}\right|^{2} & d x d t \\
& =\int_{0}^{t} \int_{\Omega}\left(\beta \varphi_{t}^{m}+\Psi^{\prime}\left(\varphi^{m}\right)+W_{, \varphi}\left(\varepsilon\left(\mathbf{u}^{m}\right), \varphi^{m}\right)\right) \Delta \varphi^{m} d x d t .
\end{array}
$$

Now, using the Cauchy-Schwarz inequality and the growth conditions (17),

$$
\left|\Psi^{\prime}(\varphi)\right| \leq c\left(|\varphi|^{3}+1\right), \quad\left|W_{, \varphi}(\varepsilon(\mathbf{u}), \varphi)\right| \leq c(|\varepsilon(\mathbf{u})|+1),
$$

it follows that

$$
\begin{aligned}
\gamma \| \Delta \varphi^{m} & \|_{L_{2}\left(\Omega^{t}\right)} \\
& \leq \beta\left\|\varphi_{t}^{m}\right\|_{L_{2}\left(\Omega^{t}\right)}+\left\|\Psi^{\prime}\left(\varphi^{m}\right)\right\|_{L_{2}\left(\Omega^{t}\right)}+\left\|W_{, \varphi}\left(\varepsilon\left(\mathbf{u}^{m}\right), \varphi^{m}\right)\right\|_{L_{2}\left(\Omega^{t}\right)} \\
& \leq \beta\left\|\varphi_{t}^{m}\right\|_{L_{2}\left(\Omega^{t}\right)}+c t^{1 / 2}\left(\left\|\varphi^{m}\right\|_{L_{\infty}\left(0, t ; L_{6}(\Omega)\right)}^{3}+\left\|\varepsilon\left(\mathbf{u}^{m}\right)\right\|_{L_{\infty}\left(0, t ; L_{2}(\Omega)\right)}+1\right) .
\end{aligned}
$$

Hence, by (29), (37) and (38), we conclude that

$$
\left\|\Delta \varphi^{m}\right\|_{L_{2}\left(\Omega^{t}\right)} \leq c t^{1 / 2} .
$$

Now, on account of the inequality

$$
\|\varphi\|_{H^{2}(\Omega)} \leq c\left(\|\Delta \varphi\|_{L_{2}(\Omega)}+\|\varphi\|_{L_{2}(\Omega)}\right),
$$

which holds true for functions satisfying the condition $\mathbf{n} \cdot \nabla \varphi=0$ on $S$, inequalities (42) and (43) imply the assertion of the lemma.

Using standard duality arguments we shall also estimate the time derivative $\mathbf{u}_{t t}^{m}$.

LEMMA 3. Let the assumptions of Lemma 1 hold, and $\mathbf{b} \in \mathbf{L}_{2}\left(\Omega^{T}\right)$. Then, for $t \in(0, T]$,

$$
\left\|\mathbf{u}_{t^{\prime} t^{\prime}}^{m}\right\|_{L_{2}\left(0, t ;\left(H_{0}(\Omega)\right)^{\prime}\right)} \leq c_{3}(t)
$$

where $c_{3}(t)=c\left(c_{0},\|\mathbf{b}\|_{\mathbf{L}_{2}\left(\Omega^{t}\right)}\right) t^{1 / 2}$. 
Proof. For $\boldsymbol{\eta} \in L_{2}\left(0, t ; \mathbf{H}_{0}^{1}(\Omega)\right)$ we test $(27)_{1}$ with $\boldsymbol{\eta}^{m}=P^{m} \boldsymbol{\eta}$ where $P^{m}$ denotes the projection defined by

$$
P^{m} \boldsymbol{\eta}=\sum_{i=1}^{m}\left(\boldsymbol{\eta}, \mathbf{v}^{i}\right)_{\mathbf{L}_{2}(\Omega)} \mathbf{v}^{i}
$$

Then using the Cauchy-Schwarz inequality, and recalling the energy estimate (29), we obtain

$$
\begin{aligned}
\left|\int_{0}^{t}\left(\mathbf{u}_{t^{\prime} t^{\prime}}^{m}, \boldsymbol{\eta}\right) d t^{\prime}\right|=\left|\int_{0}^{t}\left(\mathbf{u}_{t^{\prime} t^{\prime}}^{m}, P^{m} \boldsymbol{\eta}\right) d t^{\prime}\right| \\
=\left|\int_{0}^{t}\left[-\left(\mathbf{A} \varepsilon\left(\mathbf{u}^{m}\right), \boldsymbol{\varepsilon}\left(P^{m} \boldsymbol{\eta}\right)\right)+\left(z^{\prime}\left(\varphi^{m}\right) \mathbf{B} \nabla \varphi^{m}+\mathbf{b}, P^{m} \boldsymbol{\eta}\right)\right] d t^{\prime}\right| \\
\leq c\left(\left\|\boldsymbol{\varepsilon}\left(\mathbf{u}^{m}\right)\right\|_{\mathbf{L}_{2}\left(\Omega^{t}\right)}\left\|\nabla P^{m} \boldsymbol{\eta}\right\|_{\mathbf{L}_{2}\left(\Omega^{t}\right)}\right) \\
\quad+c\left(\left\|\nabla \varphi^{m}\right\|_{\mathbf{L}_{2}\left(\Omega^{t}\right)}+\|\mathbf{b}\|_{\mathbf{L}_{2}\left(\Omega^{t}\right)}\right)\left\|P^{m} \boldsymbol{\eta}\right\|_{\mathbf{L}_{2}\left(\Omega^{t}\right)} \\
\leq c\left(c_{0} t^{1 / 2}+\|\mathbf{b}\|_{\mathbf{L}_{2}\left(\Omega^{t}\right)}\right)\left\|P^{m} \boldsymbol{\eta}\right\|_{L_{2}\left(0, t ; \mathbf{H}^{1}(\Omega)\right)} \\
\leq c\left(c_{0},\|\mathbf{b}\|_{\mathbf{L}_{2}\left(\Omega^{t}\right)}\right) t^{1 / 2}\|\boldsymbol{\eta}\|_{L_{2}\left(0, t ; \mathbf{H}^{1}(\Omega)\right)}
\end{aligned}
$$

for any $\boldsymbol{\eta} \in L_{2}\left(0, t ; \mathbf{H}_{0}^{1}(\Omega)\right)$. This shows the assertion.

\section{Proof of Theorems 1,2}

Proof of Theorem 1. From (29), (36)-(39), (44) it follows that there exists a pair $(\mathbf{u}, \varphi)$ with

$$
\begin{aligned}
& \mathbf{u} \in L_{\infty}\left(0, T ; \mathbf{H}_{0}^{1}(\Omega)\right), \quad \mathbf{u}_{t} \in L_{\infty}\left(0, T ; \mathbf{L}_{2}(\Omega)\right), \\
& \mathbf{u}_{t t} \in L_{2}\left(0, T ;\left(\mathbf{H}_{0}^{1}(\Omega)\right)^{\prime}\right), \\
& \varphi \in L_{\infty}\left(0, T ; H^{1}(\Omega)\right) \cap L_{2}\left(0, T ; H^{2}(\Omega)\right), \quad \varphi_{t} \in L_{2}\left(\Omega^{T}\right),
\end{aligned}
$$

and a subsequence $\left(\mathbf{u}^{m}, \varphi^{m}\right)$ of solutions to $(27)$ (which we still denote by the same indices) such that

$$
\begin{array}{ll}
\mathbf{u}^{m} \rightarrow \mathbf{u} & \text { weak }^{*} \text { in } L_{\infty}\left(0, T ; \mathbf{H}_{0}^{1}(\Omega)\right), \\
\mathbf{u}_{t}^{m} \rightarrow \mathbf{u}_{t} \quad \text { weak }^{*} \text { in } L_{\infty}\left(0, T ; \mathbf{L}_{2}(\Omega)\right), \\
\mathbf{u}_{t t}^{m} \rightarrow \mathbf{u}_{t t} \quad \text { weakly in } L_{2}\left(0, T ;\left(\mathbf{H}_{0}^{1}(\Omega)\right)^{\prime}\right), \\
\varphi^{m} \rightarrow \varphi \quad \text { weak }{ }^{*} \text { in } L_{\infty}\left(0, T ; H^{1}(\Omega)\right) \text { and weakly in } L_{2}\left(0, T ; H^{2}(\Omega)\right), \\
\varphi_{t}^{m} \rightarrow \varphi_{t} \quad \text { weakly in } L_{2}\left(\Omega^{T}\right) .
\end{array}
$$

Then by the standard compactness results ([10, Corollary 4]) it follows in particular that for subsequences (still denoted by the same indices) 


$$
\begin{array}{ll}
\mathbf{u}^{m} \rightarrow \mathbf{u} & \text { strongly in } L_{2}\left(0, T ; \mathbf{L}_{q}(\Omega)\right) \cap C\left([0, T] ; \mathbf{L}_{q}(\Omega)\right) \\
& \text { for } q<6, \text { and a.e. in } \Omega^{T}, \\
\mathbf{u}_{t}^{m} \rightarrow \mathbf{u}_{t} & \text { strongly in } C\left([0, T] ;\left(\mathbf{H}_{0}^{1}(\Omega)\right)^{\prime}\right), \\
\varphi^{m} \rightarrow \varphi & \text { strongly in } L_{2}\left(0, T ; H^{1}(\Omega)\right) \cap C\left([0, T] ; L_{2}(\Omega)\right) \\
& \text { and a.e. in } \Omega^{T} .
\end{array}
$$

Hence,

$$
\begin{array}{ll}
\mathbf{u}^{m}(0)=\mathbf{u}_{0}^{m} \rightarrow \mathbf{u}(0) & \text { strongly in } \mathbf{L}_{q}(\Omega), q<6, \\
\mathbf{u}_{t}^{m}(0)=\mathbf{u}_{1}^{m} \rightarrow \mathbf{u}_{t}(0) & \text { strongly in }\left(\mathbf{H}_{0}^{1}(\Omega)\right)^{\prime}, \\
\varphi^{m}(0)=\varphi_{0}^{m} \rightarrow \varphi(0) & \text { strongly in } L_{2}(\Omega),
\end{array}
$$

which together with convergences (28) implies that

$$
\mathbf{u}(0)=\mathbf{u}_{0}, \quad \mathbf{u}_{t}(0)=\mathbf{u}_{1}, \quad \varphi(0)=\varphi_{0} .
$$

The relations (45) and (49) imply the assertion (20) of the theorem.

We introduce the weak formulation corresponding to the Faedo-Galerkin approximation (27):

$$
\begin{aligned}
& \int_{0}^{T}\left(\left\langle\mathbf{u}_{t t}^{m}, \boldsymbol{\eta}\right\rangle_{\left(\mathbf{H}_{0}^{1}(\Omega)\right)^{\prime}, \mathbf{H}_{0}^{1}(\Omega)}+\left(\mathbf{A} \varepsilon\left(\mathbf{u}^{m}\right), \boldsymbol{\varepsilon}(\boldsymbol{\eta})\right)\right) d t \\
&=\int_{0}^{T}\left(z^{\prime}\left(\varphi^{m}\right) \mathbf{B} \nabla \varphi^{m}+\mathbf{b}, \boldsymbol{\eta}\right) d t \quad \text { for any } \boldsymbol{\eta} \in E_{\mathbf{V}_{m}},
\end{aligned}
$$

$$
\begin{aligned}
& \int_{0}^{T}\left(\beta \varphi_{t}^{m}-\gamma \Delta \varphi^{m}+\Psi^{\prime}\left(\varphi^{m}\right)+W_{, \varphi}\left(\varepsilon\left(\mathbf{u}^{m}\right), \varphi^{m}\right), \xi\right) d t=0 \\
& \text { for any } \xi \in E_{W_{m}},
\end{aligned}
$$

where

$$
\begin{aligned}
& E_{\mathbf{V}_{m}}=\left\{\boldsymbol{\eta}: \boldsymbol{\eta}=\sum_{j=1}^{m} e_{j}^{m}(t) \mathbf{v}_{j}(x), e_{j}^{m} \in L_{2}(0, T), \mathbf{v}_{j} \in \mathbf{V}_{m}\right\}, \\
& E_{W_{m}}=\left\{\xi: \xi=\sum_{j=1}^{m} c_{j}^{m}(t) w_{j}(x), c_{j}^{m} \in L_{2}(0, T), w_{j} \in W_{m}\right\} .
\end{aligned}
$$

To pass to the limit as $m \rightarrow \infty$ in (50) we follow the standard procedure (see e.g. [7]). Namely, we fix $m=m_{0} \in \mathbb{N}$ in the spaces of test functions $\boldsymbol{\eta}, \xi$, and take subsequences (46) with $m \geq m_{0}$.

Clearly, by the weak convergences (46) the linear terms in (50) converge to the corresponding limits.

Thus, it remains to examine the convergence of the nonlinear terms $z^{\prime}\left(\varphi^{m}\right) \mathbf{B} \nabla \varphi^{m}, \Psi^{\prime}\left(\varphi^{m}\right)$ and $W_{, \varphi}\left(\varepsilon\left(\mathbf{u}^{m}\right), \varphi^{m}\right)$. Recalling the growth conditions (17), and using the energy bounds (29), (37) it follows that 


$$
\begin{aligned}
\left\|z^{\prime}\left(\varphi^{m}\right) \mathbf{B} \nabla \varphi^{m}\right\|_{L_{\infty}\left(0, T ; L_{2}(\Omega)\right)} & \leq c\left\|\nabla \varphi^{m}\right\|_{L_{\infty}\left(0, T ; L_{2}(\Omega)\right)} \leq c c_{0}, \\
\left\|\Psi^{\prime}\left(\varphi^{m}\right)\right\|_{L_{\infty}\left(0, T ; L_{2}(\Omega)\right)} & \leq c\left(\left\|\varphi^{m}\right\|_{L_{\infty}\left(0, T ; L_{6}(\Omega)\right)}^{3}+1\right) \leq c, \\
\left\|W_{, \varphi}\left(\varepsilon\left(\mathbf{u}^{m}\right), \varphi^{m}\right)\right\|_{L_{\infty}\left(0, T ; L_{2}(\Omega)\right)} & \leq c\left(\left\|\varepsilon\left(\mathbf{u}^{m}\right)\right\|_{L_{\infty}\left(0, T ; L_{2}(\Omega)\right)}+1\right) \leq c .
\end{aligned}
$$

Thanks to the uniformity in $m$ of these estimates and the pointwise convergences (47) we can apply the standard nonlinear convergence lemma (see [6, Chap. 1, Lemma 1.3]) to conclude that

$$
\begin{aligned}
& z^{\prime}\left(\varphi^{m}\right) \mathbf{B} \nabla \varphi^{m} \rightarrow z^{\prime}(\varphi) \mathbf{B} \nabla \varphi \quad \text { weak }^{*} \text { in } L_{\infty}\left(0, T ; \mathbf{L}_{2}(\Omega)\right), \\
& \Psi^{\prime}\left(\varphi^{m}\right)=2\left(\varphi^{m}\right)^{3}-3\left(\varphi^{m}\right)^{2}+\varphi^{m} \rightarrow 2 \varphi^{3}-3 \varphi^{2}+\varphi=\Psi^{\prime}(\varphi) \\
& \text { weak }^{*} \text { in } L_{\infty}\left(0, T ; L_{2}(\Omega)\right) \text {, } \\
& W_{, \varphi}\left(\boldsymbol{\varepsilon}\left(\mathbf{u}^{m}\right), \varphi^{m}\right)=z^{\prime}\left(\varphi^{m}\right)\left(\mathbf{B} \cdot \boldsymbol{\varepsilon}\left(\mathbf{u}^{m}\right)+D z\left(\varphi^{m}\right)+E\right) \\
& \rightarrow z^{\prime}(\varphi)(\mathbf{B} \cdot \varepsilon(\mathbf{u})+D z(\varphi)+E)=W_{, \varphi}(\varepsilon(\mathbf{u}), \varphi) \\
& \text { weak }^{*} \text { in } L_{\infty}\left(0, T ; L_{2}(\Omega)\right) \text {. }
\end{aligned}
$$

Consequently, passing to the limit in (50) for a subsequence $m_{0} \leq m$ $\rightarrow \infty$, we conclude that the identities in Theorem 1 are satisfied for all test functions $\boldsymbol{\eta} \in E_{\mathbf{V}_{m_{0}}}, \xi \in E_{W_{m_{0}}}$.

Next, letting $m_{0} \rightarrow \infty$, by density arguments we arrive at identities (21). Clearly, the a priori estimates (22) are consequences of the uniform estimates in Lemmas 1-3 and the weak convergences (46). This proves the theorem.

Proof of Theorem 2. Let $F(t)$ denote the total energy of the system,

$$
F(t)=\int_{\Omega}\left[\frac{1}{2}\left|\mathbf{u}_{t}\right|^{2}+W(\varepsilon(\mathbf{u}), \varphi)+\Psi(\varphi)+\frac{1}{2} \gamma|\nabla \varphi|^{2}\right] d x .
$$

By the same arguments as in Lemma 1 we can show that solutions of problem (1)-(2) satisfy the following energy identity in the distribution sense on the interval $(0, T)$ :

$$
\frac{d F(t)}{d t}+\beta \int_{\Omega} \varphi_{t}^{2} d x=\int_{\Omega} \mathbf{b} \cdot \mathbf{u}_{t} d x .
$$

Further, from (55) it follows that (cf. (35))

$$
\begin{aligned}
\frac{1}{4}\left\|\mathbf{u}_{t}(t)\right\|_{\mathbf{L}_{2}(\Omega)}^{2} & +c_{f}\left(\|\varepsilon(\mathbf{u}(t))\|_{\mathbf{L}_{2}(\Omega)}^{2}+\|\varphi(t)\|_{L_{4}(\Omega)}^{4}+\|\nabla \varphi(t)\|_{\mathbf{L}_{2}(\Omega)}^{2}\right)-c_{f}^{\prime} \\
& \leq F(t)+\beta \int_{0}^{t}\left\|\varphi_{t}\right\|_{L_{2}(\Omega)}^{2} d t^{\prime} \leq F(0)+\|\mathbf{b}\|_{L_{1}\left(0, T ; \mathbf{L}_{2}(\Omega)\right)}^{2} .
\end{aligned}
$$

From (55) we deduce the continuity of $F(t)$ because by estimate $(22)_{1}$, using 
$\|\mathbf{b}\|_{\mathbf{L}_{2}\left(\Omega^{T}\right)} \leq c$, we get

$$
\left|F\left(t^{\prime}\right)-F\left(t^{\prime \prime}\right)\right| \leq c_{0}\left|t^{\prime}-t^{\prime \prime}\right|^{1 / 2} \int_{t^{\prime \prime}}^{t^{\prime}}\|\mathbf{b}\|_{\mathbf{L}_{2}(\Omega)}^{2} d t \leq c\left|t^{\prime}-t^{\prime \prime}\right|^{1 / 2}
$$

for $\left|t^{\prime}-t^{\prime \prime}\right|$ small enough. Moreover, (56) implies that for any $k \in \mathbb{N} \cup\{0\}$,

$$
\begin{aligned}
\left\|\mathbf{u}_{t}(k T)\right\|_{\mathbf{L}_{2}(\Omega)}^{2}+\|\mathbf{u}(k T)\|_{\mathbf{H}^{1}(\Omega)}^{2}+ & \|\varphi(k T)\|_{\mathbf{H}^{1}(\Omega)}^{2} \\
& \left.\leq c(F(0))+\|\mathbf{b}\|_{L_{1}\left(\mathbb{R}_{+} ; L_{2}(\Omega)\right)}^{2}+c_{f}^{\prime}\right) .
\end{aligned}
$$

Hence, the local solution from Theorem 1 can be prolonged step by step on the intervals $[k T,(k+1) T$ up to $k=\infty$.

\section{References}

[1] E. Fried and G. Grach, An order-parameter based theory as a regularization of a sharp-interface theory for solid-solid phase transitions, Arch. Ration. Mech. Anal. 138 (1997), 355-404.

[2] E. Fried and M. E. Gurtin, Dynamic solid-solid transitions with phase characterized by an order parameter, Phys. D 72 (1994), 287-308.

[3] Z. Kosowski, Fried-Gurtin model of transition in solids, 1-D case, Biuletyn WAT 52 (2003), no. $7,53-75$.

[4] - Unique global solvability of 1D Fried-Gurtin model, Appl. Math. (Warsaw), to appear.

[5] Z. Kosowski and I. Pawłow, Unique global solvability of Fried-Gurtin model of phase transitions in solids, Topol. Methods Nonlinear Anal. 24 (2004), 209-237.

[6] J.-L. Lions, Quelques méthodes de résolution des problèmes aux limites non linéaires, Dunod, Paris, 1969

[7] J.-L. Lions and E. Magenes, Non-Homogeneous Boundary Value Problems and Applications, Vol. I, II, Springer, Berlin, 1972.

[8] I. Pawłow and W. M. Zajączkowski, On diffused-interface models of shape memory alloys, Control Cybernet. 32 (2003), 629-658.

[9] J. Sikora, J. P. Cusumano and W. A. Jester, Spatially periodic solutions to a $1 \mathrm{D}$ model of phase transition with order parameter, Phys. D 121 (1998), 275-294.

[10] J. Simon, Compact sets in the space $L^{p}(0, T ; B)$, Ann. Mat. Pura Appl. 146 (1987), 65-97.

Institute of Mathematics and Cryptology

Faculty of Cybernetics

Military University of Technology

S. Kaliskiego 2

00-908 Warszawa, Poland

E-mail: zenekko@iname.com 